\title{
Pisatin resistance in Dictyostelium discoideum and Neurospora crassa: comparison of mutant phenotypes
}

\author{
K. G. Papavinasasundaram and Durgadas P. Kasbekar* \\ Centre for Cellular and Molecular Biology, Hyderabad 500 007, India
}

(Received 14 May 1993; revised 23 July 1993; accepted 16 August 1993)

\begin{abstract}
The pea phytoalexin pisatin, at its inhibitory concentration, was shown to have two distinct inhibitory effects on amoebae of the cellular slime mould Dictyostelium discoideum. One effect was cytolytic and was demonstrable even in non-growing cells whereas the second effect was observed only under conditions favourable to growth. Pretreatment with a sublethal concentration of pisatin induced the amoebae to acquire resistance to both these effects. Mutations in nys $C$ that alter membrane sterols and confer resistance to the polyene antibiotics nystatin and pimaricin blocked resistance to the growth-associated inhibitory effect but did not affect acquisition of resistance to the cytolytic effect. The nys $B$ sunD double mutant $H K 412$ displayed a partially constitutive resistance to the cytolytic effect but, like the nys $C$ mutants, was blocked in the acquisition of resistance to the growth-associated inhibitory effect. Pisatin-treated cells incubated in pisatin-free medium lost their ability to grow on pisatincontaining medium much more rapidly than they lost resistance to the cytolytic effect of pisatin. These results suggest that the induction of pisatin resistance may involve the turning-on of independent resistance mechanisms against the two inhibitory effects of pisatin. This could account for our inability to isolate pisatin-resistant mutants in a single step. The Neurospora crassa erg1 and erg 3 mutants that have altered membrane sterols and are nystatin resistant displayed sensitivity to pisatin. The pisatin-sensitivity phenotype of the erg mutants was used in selections to identify complementing plasmids from an ordered Neurospora genomic library. The association of pisatin sensitivity with membrane sterol alterations in both $D$. discoideum and $N$. crassa supports the hypothesis that mechanisms underlying nondegradative pisatin resistance are evolutionarily conserved.
\end{abstract}

\section{Introduction}

Phytoalexins are antimicrobial compounds produced by plants, possibly to prevent microbial infection. Pisatin (6a-hydroxy-3-methoxy-8,9-methylenedioxy pterocarpan) is the major isoflavonoid phytoalexin of pea (Pisum sativum $\mathrm{L}$.). We have previously reported that wild-type amoebae of the cellular slime mould Dictyostelium discoideum can be induced to acquire a nondegradative resistance to an inhibitory concentration of pisatin when grown on a medium containing a sublethal concentration $\left(50 \mu \mathrm{g} \mathrm{ml}^{-1}\right)$ (Kasbekar \& Papavinasasundaram, 1992). When plated on medium containing $150 \mu \mathrm{g}$ pisatin $\mathrm{ml}^{-1}$, the induced amoebae displayed a greater than $10^{4}$-fold increase in plating efficiency compared to uninduced amoebae derived from pisatin-free medium. Maintenance of resistance required the continued presence of pisatin in the medium because pisatin-resistant cells

${ }^{*}$ Author for correspondence. Tel. +91842 852241; fax +91842 851195; e-mail Kas\%ccmb@uunet.in. reverted rapidly during passage on pisatin-free medium. Inducible nondegradative pisatin resistance has previously been reported for the pea pathogenic fungus Nectria haematococca Berk. and Br. mating population VI (imperfect form: Fusarium solani) (Denny \& VanEtten, 1983) but efforts to elucidate its mechanism have not been very successful (Denny et al., 1987). Since $D$. discoideum is a more tractable system for genetic and molecular studies than most phytopathogenic fungi, we have used it as a model system to study nondegradative pisatin resistance in the expectation that cellular slime moulds and fungi may share the same resistance mechanism.

We found that an alteration of membrane sterol composition, either with the azasterol A25822B or by mutations in $n y s C$ that confer resistance to the polyene antibiotics nystatin and pimaricin, suppressed the induction of pisatin resistance in $D$. discoideum (Kasbekar \& Papavinasasundaram, 1992). Induction was also suppressed in the nys $B$ sunD double mutant strain HK412 (Kasbekar \& Bhavani Prasanna, 1992). HK412 
was isolated in screens for suppressors of nys $B$ (K. Lionetti \& E. R. Katz, unpublished). Mutations in nys $B$ also alter membrane sterols and confer nystatin resistance (Scandella et al., 1980) but unlike the nysC mutations, they display the wild-type phenotype on pisatin (Kasbekar \& Papavinasasundaram, 1992). The nys $B$ sunD double mutant has the same sterol composition as the $n y s B$ parent (K. Lionetti \& E. R. Katz, unpublished) which suggests that the sunD mutation defines an additional requirement, perhaps another membrane component, for pisatin resistance.

In spite of the ease with which pisatin resistance can be induced in the wild-type, and in contrast to the readiness with which multiple mutations have been identified that suppress the induction phenomenon, we have not been able to isolate constitutive pisatin-resistant mutants by direct selection on inhibitory concentrations. One possible explanation for this result is that pisatin has more than one inhibitory effect at its inhibitory concentration and that the induction of resistance involves the turningon of distinct and independent resistance mechanisms. Consequently, a single gene mutation would not simultaneously turn on all the mechanisms required for growth on pisatin medium and would thereby account for our inability to obtain pisatin-resistant mutants in a single step. If this model is correct, we might expect the $n y s C$ and the nys $B$ sunD mutants to be blocked in some, but not all, of the pisatin-induced resistance mechanisms, that is, they might show a partial induction of resistance during growth on the sublethal concentration. In this paper, we present results of experiments designed to test this possibility. We interpret our results to suggest that pisatin inhibits amoebae by two distinct inhibitory effects; a cytolytic effect and an additional inhibitory effect that is expressed only during growth. Further, we demonstrate that the mutations block the acquisition of resistance to only the latter effect but do not affect the acquisition of resistance to the cytolytic effect.

Additionally, we have found that sterol mutations in Neurospora crassa also confer sensitivity to pisatin but there is a significant difference in the pisatin-sensitivity phenotype of the sterol mutants in Neurospora from that in Dictyostelium. The model for the involvement of independent mechanisms induced against the inhibitory effects of pisatin also provides a simple explanation for the differences in the pisatin-sensitivity phenotypes in the two systems.

\section{Methods}

Strains. All the $D$. discoideum strains used were derivatives of $D$. discoideum NC4 (Raper, 1935) and have been described previously (Scandella et al., 1980; Kasbekar \& Bhavani Prasanna, 1992). N. crassa strains 988 (ORa), 2721 (ergla) and 2725 (erg3a) were obtained from the Fungal Genetics Stocks Center (FGSC), Department of Micro- biology, University of Kansas Medical Center, Kansas City, KS USA, and also from Dr Morris Grindle, University of Sheffield, UK.

Pisatin extraction. Pisatin was extracted from germinated pea seeds (Pisum sativum L) following published procedures (Sweigard \& VanEtten, 1987).

Media and growth conditions. D. discoideum amoebae were grown on lawns of Enterobacter aerogenes at $22^{\circ} \mathrm{C}$ on SM agar plates (Sussman, 1966). A stock solution $\left(10 \mathrm{mg} \mathrm{m}^{-1}\right)$ of pisatin (pis) in dimethyl sulphoxide (DMSO) was used to supplement SM agar plates just before the latter were poured. The concentrations used are indicated in the abbreviations SM-pis50 $\left(50 \mu \mathrm{g} \mathrm{m}^{-1}\right)$ and SM-pis150 $\left(150 \mu \mathrm{g} \mathrm{ml}^{-1}\right)$.

Bonner's salt solution (BSS) (Bonner, 1947) was used for suspending $D$. discoideum amoebae to make serial dilutions for determining the efficiencies of plating (EOP), and to incubate the amoebae for investigating the induction and the loss of pisatin resistance. Wherever pisatin was used in the treatments, an equal volume of DMSO was included in the controls. At the concentrations used, DMSO had no effect on cell viability. Routinely, more than $95 \%$ of the cells estimated from a haemocytometer count produced plaques when plated on SM medium.

Mutagenesis of $D$. discoideum amoebae was carried out following the procedure of Loomis (1987), with the modification that the amoebae were treated with $500 \mu \mathrm{g} \quad N$-methyl- $N^{\prime}$-nitro- $N$-nitrosoguanidine (MNNG) $\mathrm{ml}^{-1}$ for $5 \mathrm{~min}$. In control experiments, we obtained a 30 -fold increase in the frequency of mutants resistant to growth on medium containing $3 \%(\mathrm{v} / \mathrm{v})$ methanol (frequency of methanol-resistant mutants before and after MNNG treatment was $8 \times 10^{-6}$ and $2.4 \times 10^{-4}$ per viable cell, respectively). This indicated that the mutagen treatment was effective.

The $N$. crassa strains were maintained at room temperature on Vogel's minimal medium (Davis \& de Serres, 1970) supplemented with $1.5 \%(\mathrm{w} / \mathrm{v})$ sucrose, or on glucose-peptone (GP) medium (10 g glucose, $5 \mathrm{~g}$ Oxoid peptone, $1 \mathrm{~g} \mathrm{KH}_{2} \mathrm{PO}_{4}, 0.5 \mathrm{~g} \mathrm{MgSO}_{4} .7 \mathrm{H}_{2} \mathrm{O}$, in 1 litre distilled water).

Inhibition of protein synthesis. $D$. discoideum amoebae were suspended (approx. density $10^{7} \mathrm{ml}^{-1}$ ) in BSS, and in BSS containing $200 \mu \mathrm{g}$ cycloheximide (Sigma) $\mathrm{ml}^{-1}$, and incubated on a shaker at $22^{\circ} \mathrm{C}$. After $30 \mathrm{~min}, 10 \mu \mathrm{Ci} \mathrm{m}^{-1} \quad\left(370 \mathrm{kBq} \mathrm{ml}^{-1}\right) \quad\left[{ }^{35} \mathrm{~S}\right]$ methionine $\left[1094 \mathrm{Ci} \mathrm{mmol}^{-1}\left(4.048 \times 10^{10} \mathrm{kBq} \mathrm{mmol}^{-1}\right)\right]$ and $0.3 \mu \mathrm{M}$ carrier-methionine were added and incubated for a further $4 \mathrm{~h}$. Incorporation of the label was stopped by the addition of excess $\left(10^{4}\right.$-fold) carriermethionine, and the amoebae were lysed in BSS containing $0.1 \%$ SDS. An aliquot of cell lysate was precipitated onto Whatman GF/C discs with $10 \%(\mathrm{w} / \mathrm{v})$ trichloroacetic acid (TCA) containing $0.1 \mathrm{M}$ carriermethionine. TCA-soluble material was removed by washing the filters twice at $25^{\circ} \mathrm{C}$ and once at $70{ }^{\circ} \mathrm{C}$ using $5 \%(\mathrm{w} / \mathrm{v})$ TCA containing $0.05 \mathrm{M}$ carrier-methionine. The filters were then soaked briefly in ethanol, dried, and the incorporation of label in the TCA-insoluble fraction was determined by counting in a toluene-based scintillation fluid.

Cloning of the Neurospora erg1 and erg 3 genes. Twenty-three pools of plasmid DNA were prepared, each representing two or three 96-well microtitre plates of the 50-plate Orbach/Sachs Neurospora genome library (obtained from the FGSC). The plasmid pools were used to transform protoplasts prepared from ergl and erg3 mutant strains. Published protocols were followed to prepare the protoplasts (Vollmer \& Yanofsky, 1986) and for transformation (Sellitrennikoff \& Sachs, 1991). Double selection on Vogel's medium supplemented with hygromycin and pisatin was used to identify the pools that harboured plasmid DNA capable of yielding pisatin-resistant transformants. These plasmids presumably contained the corresponding wild-type alleles. The identified pools were subdivided into subpools and the 
Table 1. Induction of pisatin resistance in wild-type and mutant D. discoideum

The EOPs on SM agar were calculated as the ratio of the number of plaques on SM agar following the BSS-pis150 treatment to the number following the BSS-DMSO control treatment. The EOPs on SM-pis 150 were calculated as the ratio of the number of plaques on SM-pis150 agar to that on pisatin-free SM agar.

\begin{tabular}{lccccc}
\hline \hline & & \multicolumn{2}{c}{$\begin{array}{c}\text { EOP on SM after incubation } \\
\text { in BSS-pis150* }\end{array}$} & $\begin{array}{c}\text { EOP on } \\
\text { SM-pis150 } \\
\text { after }\end{array}$ \\
\cline { 3 - 5 } Strain & $\begin{array}{c}\text { Relevant } \\
\text { genotype }\end{array}$ & Uninduced & $\begin{array}{c}\text { Induced on } \\
\text { SM-pis50 }\end{array}$ & $\begin{array}{c}\text { Induced in } \\
\text { BSS-pis50 }\end{array}$ & $\begin{array}{c}\text { incubation in } \\
\text { BSS-pis50 }\end{array}$ \\
\hline DdB & + & $<5 \times 10^{-6}$ & 1.0 & 0.37 & 0.52 \\
M28 & + & $4 \times 10^{-5}$ & 0.30 & 0.5 & 0.02 \\
TS12M & + & $3.6 \times 10^{-5}$ & 0.83 & 0.5 & 0.05 \\
HK10 & nysC208 & $2 \times 10^{-5}$ & 0.40 & 1.0 & $<10^{-5}$ \\
HK12 & nysC210 & $8 \times 10^{-5}$ & 0.68 & 1.0 & $9 \times 10^{-6}$ \\
HK412 & nysB sunD & $3.7 \times 10^{-3}$ & 1.0 & 1.0 & $<1.4 \times 10^{-5}$ \\
\hline \hline
\end{tabular}

*Amoebae (approx. $5 \times 10^{6} \mathrm{ml}^{-1}$ ) were incubated in BSS-pis 150 and in BSS-DMSO. After $5 \mathrm{~h}$ incubation, the amoebae were plated on SM agar to determine the viable counts.

$\dagger$ Amoebae (approx. $5 \times 10^{6} \mathrm{ml}^{-1}$ ) were incubated in BSS-pis50 for $4 \mathrm{~h}$.

process was iterated until subpools representing single clones were identified. This approach is known as cloning by sib selection (Vollmer $\&$ Yanofsky, 1986). The selection conditions were $40 \mu \mathrm{g}$ pisatin $\mathrm{ml}^{-1}$ in the top and bottom agar and $200 \mu \mathrm{g}$ hygromycin $\mathrm{ml}^{-1}$ in the bottom agar.

\section{Results}

Incubation in BSS-pis150 is cytocidal

To determine whether pisatin has a cytocidal effect on the amoebae, we examined whether the amoebae lost viability during incubation in BSS containing $150 \mu \mathrm{g}$ pisatin $\mathrm{ml}^{-1}$ (BSS-pis150). BSS is a non-nutrient medium which ordinarily allows amoebae to maintain viability for more than $24 \mathrm{~h}$. If pisatin induces cell death, the EOP upon transfer of the amoebae from BSS-pis150 to SM medium should decrease compared to that of amoebae incubated in a BSS-DMSO control. We found that after a $5 \mathrm{~h}$ incubation in BSS-pis 150 , the EOP of the amoebae had dropped to approximately $10^{-5}$ (Table 1). Examination under a microscope revealed that incubation in BSS-pis150 induced the amoebae to swell and lyse. The addition of $0.5 \mathrm{M}$-sucrose to the BSS-pis 150 protected against the pisatin-induced lysis and loss of viability (EOP after a $5 \mathrm{~h}$ incubation of DdB amoebae in BSSpis 150 with $0 \cdot 5 \mathrm{M}$-sucrose was $0 \cdot 1$ ). As anticipated, wildtype amoebae induced to become pisatin-resistant by growth on SM medium containing a sublethal concentration of pisatin (SM-pis50) (Kasbekar \& Papavinasasundaram, 1992) were resistant in this assay (Table 1). These experiments demonstrated that at its inhibitory concentration $\left(150 \mu \mathrm{g} \mathrm{ml}^{-1}\right)$, pisatin has a cytolytic effect that is mediated perhaps by a loss in osmotic control due to a permeability change of the cytoplasmic membrane, and that the amoebae acquired resistance to this effect during growth on medium containing a sublethal concentration of pisatin (SM-pis50).

\section{Evidence for an additional inhibitory effect of pis 150}

We examined whether the block in the acquisition of pisatin resistance in nys $C$ and the nys $B$ sunD mutants could be explained by their inability to acquire resistance to the cytolytic effect. We found, however, that growth on SM-pis50 medium (or pretreatment in BSS-pis50, see below) induced the mutant amoebae to acquire resistance to the cytolytic effect of pisatin as effectively as the wildtype (Table 1). These results indicated that the acquisition of resistance to lysis by pisatin in the assay above was not sufficient to support growth on SMpis 150 . One model suggested by this result is that in addition to exerting a cytolytic effect, pis 150 also inhibits amoebae by an additional effect. Consequently, for growth on SM-pis150, the amoebae have to acquire resistance to both the inhibitory effects of pisatin. In this model, the $n y s C$ and the nys $B$ sun $D$ mutants can acquire resistance to the cytolytic effect as effectively as the wildtype but are blocked in the acquisition of resistance to the second inhibitory effect.

An alternative model that is compatible with the above results is that pisatin has only one inhibitory effect (i.e. cytolysis), and that the acquisition of resistance to this effect is indeed sufficient for pisatin resistance in the wildtype, but that in the mutants the combination of two different membrane alterations, one pisatin-induced and the other mutation-induced, in some way inhibits growth. To distinguish between these models, we did experiments to determine whether the resistance to the cytolytic effect 


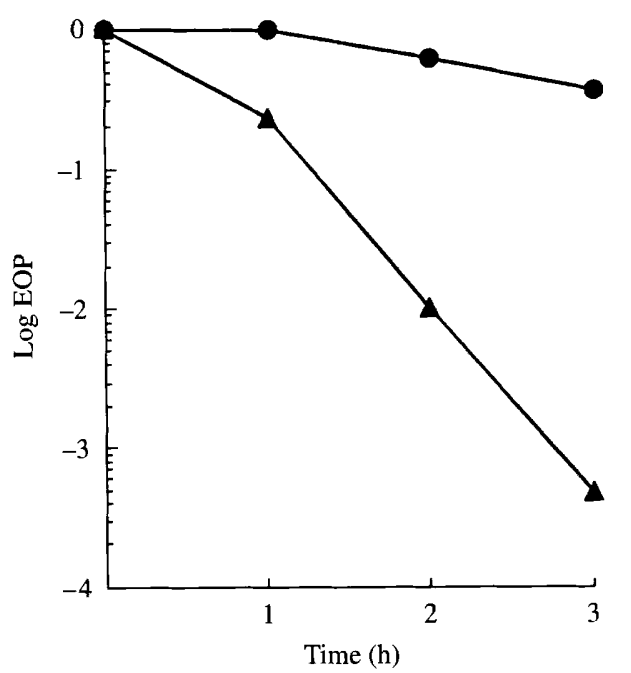

Fig. 1. Kinetics of loss of pisatin resistance in wild-type amoebae. DdB amoebae $\left(1.6 \times 10^{6} \mathrm{ml}^{-1}\right)$ derived from SM-pis150 medium were incubated in BSS for $3 \mathrm{~h}$. At different time-points during this incubation, aliquots of the suspension were assayed for EOP on SMpis150 ( $\mathbf{\Delta})$ by determining the ratio of the number of plaques on SMpis 150 agar to that on pisatin-free SM agar. At the same time-points, the amoebae were also assayed for resistance to cytolysis during a $4 \mathrm{~h}$ incubation in BSS-pis150 ( ) . In this experiment, the EOP refers to the ratio of the number of plaques on SM agar following the BSS-pis 150 treatment to the number following the BSS-DMSO control treatment. Data are plotted on a log scale relative to the $0 \mathrm{~h}$ value.

could be dissociated from competence to grow on SMpis 150 , even in the wild-type. We found that wild-type amoebae that were induced to become pisatin-resistant lost their resistance phenotype after a $4 \mathrm{~h}$ incubation in pisatin-free BSS. However, this loss in resistance appeared to occur in a stepwise manner; the ability to grow on SM-pis 150 was lost considerably faster than the resistance to lysis by BSS-pis150. After a $3 \mathrm{~h}$ incubation in BSS, the EOP of pisatin-resistant wild-type amoebae on SM-pis 150 had dropped by four orders of magnitude but there was only a $63 \%$ reduction in their resistance to the cytolytic effect (Fig. 1). This demonstrated that resistance to the cytolytic effect is not sufficient for growth on SM-pis 150, even in the wild-type. This result argues against the second model (that acquisition of resistance to the cytolytic effect is sufficient for the growth of the wild-type on SM-pis150), and therefore supports the first model (that amoebae have to acquire resistance to the cytolytic and the additional inhibitory effect of pisatin in order to be able to grow on SMpis150).

It is noteworthy that SM-grown (that is, uninduced) HK412 amoebae were generally more resistant to the cytolytic effect of pisatin than similarly grown wild-type amoebae (Table 1). However, the two populations of amoebae are equally sensitive to growth on SM-pis 150
(Kasbekar \& Bhavani Prasanna, 1992). These results rule out the possibility that resistance to the two inhibitory effects reflects two thresholds of a single mechanism and suggest instead that the mechanisms that confer resistance to the two inhibitory effects are independently induced.

To determine the nature of the second inhibitory effect of pisatin we examined the fate of SM-pis50-derived nys $C$ mutant cells following their plating on SM-pis 150 medium. At various time-points following the transfer, the cell viability was determined by harvesting cells and bacteria from approximately equal areas of the SMpis150 agar surface and replating on drug-free SM medium. Even after $24 \mathrm{~h}$ on SM-pis $150,20 \%$ of the HK10 cells remained viable, that is they were capable of forming plaques on SM medium. Comparable results were obtained for the strain HK12. Thus, in contrast to the cytolytic effect, growth inhibition of induced nysC cells on SM-pis 150 is not due to rapid cell death. This result suggests that inhibition by pisatin of induced nys $C$ cells represents a cytostatic effect. The fivefold decrease in cell viability that was observed over the duration of the experiment may reflect a secondary toxic effect of rapidly growing bacteria on non-dividing amoebae. In order to distinguish this inhibitory effect of pisatin from the cytolytic effect, we refer to it as the growth-associated inhibition.

\section{Induction of pisatin resistance in BSS-pis50}

In a previous report (Kasbekar \& Papavinasasundaram, 1992), wild-type amoebae were shown to acquire pisatin resistance during growth on SM-pis50. We have now tested whether amoebae could acquire the pisatinresistance phenotype during incubation in BSS-pis 50 . Table 1 summarizes the results of these experiments and shows that a $4 \mathrm{~h}$ incubation in BSS-pis 50 induced the wild-type amoebae, but not the nys $C$ mutants nor the $n y s B$ sun $D$ mutant, to acquire the pisatin-resistance phenotype (i.e. the ability to grow on SM-pis150).

Since a $4 \mathrm{~h}$ incubation in BSS-pis50 can induce pisatin resistance, and pisatin-resistant amoebae are not killed by a $5 \mathrm{~h}$ incubation in BSS-pis 150 , we subjected amoebae to both the treatments in a sequential manner (i.e. a $4 \mathrm{~h}$ incubation in BSS-pis 50 followed by a $5 \mathrm{~h}$ incubation in BSS-pis150). Following the sequential treatment, the amoebae were plated on SM medium to determine the EOP. The result (Table 1) shows that the wild-type, as well as the nys $C$ and the nys $B$ sunD mutants, acquired resistance to the cytolytic effect of pisatin. The amoebae were also plated on SM-pis150 following the BSS-pis 150 incubation and, as expected, the wild-type strains were resistant whereas the mutant strains were sensitive to growth on the phytoalexin-containing medium (data not 
Table 2. Induction of pisatin resistance involves active metabolic responses requiring protein synthesis

SM-derived DdB amoebae (approx. $10^{6} \mathrm{ml}^{-1}$ ) were pretreated in BSS for $4 \mathrm{~h}$ at $22{ }^{\circ} \mathrm{C}$ unless otherwise indicated. The EOPs were calculated as the ratio of the number of plaques on SM-pis150 agar to that on pisatin-free SM agar.

\begin{tabular}{lc}
\hline \multicolumn{1}{c}{ Pretreatment } & EOP on \\
SM-pis150
\end{tabular}

* Cycloheximide was used at $200 \mu \mathrm{g} \mathrm{ml}^{-1}$.

Table 3. Loss of pisatin resistance involves active metabolic responses requiring protein synthesis

SM-pis50 derived DdB amoebae (approx. $10^{6} \mathrm{ml}^{-1}$ ) were incubated in BSS for $4 \mathrm{~h}$ as indicated. The EOPs were calculated as the ratio of the number of plaques on SM-pis150 agar to that on pisatin-free SM agar.

\begin{tabular}{cc}
\hline \hline & EOP on \\
Pretreatment & SM-pis150 \\
\hline $22{ }^{\circ} \mathrm{C}$ & $9 \times 10^{-6}$ \\
+ Cycloheximide* & 0.54 \\
$4{ }^{\circ} \mathrm{C}$ & 0.7 \\
\hline \hline
\end{tabular}

* Cycloheximide was used at $200 \mu \mathrm{g} \mathrm{ml}^{-1}$.

shown). These results support our conclusion that nys $C$ and the nysB sunD mutations do not impair the acquisition of resistance to the cytolytic effect of pisatin even though they block the acquisition of resistance to growth on SM-pis150 (and therefore to its growthassociated inhibition).

Acquisition and loss of pisatin resistance involve active metabolic responses requiring protein synthesis

The induction of pisatin resistance in the wild-type DdB amoebae in BSS-pis50 was blocked if the incubation temperature was lowered to $4{ }^{\circ} \mathrm{C}$ or by the addition of the cytoplasmic-translation-inhibitor cycloheximide $\left(200 \mu \mathrm{g} \mathrm{ml}^{-1}\right.$; Table 2). Likewise, the loss of pisatin resistance during incubation of resistant amoebae in BSS was blocked by incubation at $4{ }^{\circ} \mathrm{C}$ or by the addition of cycloheximide at $200 \mu \mathrm{g} \mathrm{ml}^{-1}$ (Table 3). These results indicate that both the induction and the loss of pisatin resistance involve active metabolic responses requiring protein synthesis. At the concentration used, cycloheximide blocked protein synthesis by $50 \%$ (as measured by the incorporation of $\left[{ }^{35} \mathrm{~S}\right] \mathrm{methionine}$ into the TCAprecipitable material) but this treatment had no effect on cell viability. Post-treatment viability was determined by plating the amoebae on drug-free medium (EOP on SM was $1 \cdot 0)$.

To determine whether cycloheximide blocked the acquisition of resistance to the cytolytic effect of pisatin, we incubated the wild-type DdB amoebae in BSS-pis50 together with cycloheximide $\left(200 \mu \mathrm{g} \mathrm{ml}^{-1}\right)$ for $4 \mathrm{~h}$; the amoebae were then washed in BSS and subjected to an additional $5 \mathrm{~h}$ incubation in BSS-pis150. The EOP on SM of DdB amoebae after the second treatment was $2.5 \times 10^{-5}$. This value is about four orders of magnitude lower than that obtained for amoebae pretreated in BSSpis50 without cycloheximide. This result suggests that protein synthesis is required for the acquisition of resistance to the cytolytic effect.

Table 4. Complementation in the erg1 and erg3 transformants of $N$. crassa

\begin{tabular}{|c|c|c|c|c|c|c|c|}
\hline \multirow[b]{3}{*}{ Strain } & \multirow{3}{*}{$\begin{array}{l}\text { Relevant } \\
\text { genotype }\end{array}$} & \multirow{3}{*}{$\begin{array}{l}\text { Growth } \\
\text { period } \\
\text { (h) }\end{array}$} & & & & \multicolumn{2}{|c|}{$\begin{array}{l}\text { Percentage } \\
\text { inhibition by: }\end{array}$} \\
\hline & & & \multicolumn{3}{|c|}{ Mycelial diameter $(\mathrm{mm})^{*}$} & \multirow{2}{*}{$\begin{array}{c}\text { Pisatin } \\
(40 \mu \mathrm{g} \\
\left.\mathrm{ml}^{-1}\right)\end{array}$} & \multirow{2}{*}{$\begin{array}{c}\text { Nystatin } \\
(2 \mu \mathrm{g} \\
\left.\mathrm{ml}^{-1}\right)\end{array}$} \\
\hline & & & GP & GP-pis40 & GP-nys2 & & \\
\hline ORa & + & 13 & 50 & 19 & 0 & 62 & 100 \\
\hline 2721 & erg1 & 20 & 41 & $2 \cdot 3$ & 34 & 94 & 17 \\
\hline $2721 / \mathrm{G} 17 \mathrm{~F} 12^{\dagger}$ & ergl/ $\mathrm{erg}^{+}$ & 14 & 48 & 11 & 0 & 77 & 100 \\
\hline 2725 & erg 3 & 31 & 45 & 3 & 25 & 93 & 44 \\
\hline $2725 /$ G18A10 $\dagger$ & erg3/ erg $^{+}$ & 28 & 48 & 15 & 4 & 69 & 92 \\
\hline
\end{tabular}

* Growth was calculated by measuring the mycelial diameter and subtracting the diameter of the inoculating plug $(4 \mathrm{~mm})$. As the growth rate varied between the wild-type and mutant strains, the measurements were taken when the colony diameter reached $50 \mathrm{~mm}$ on GP medium. Data represent means of four replicates. Symbols: GP-pis40, GP medium supplemented with $40 \mu \mathrm{g}$ pisatin $\mathrm{ml}^{-1}$; GP-nys2, GP medium supplemented with $2 \mu \mathrm{g}$ nystatin $\mathrm{ml}^{-1}$.

$\dagger$ Mutant protoplasts transformed with the corresponding complementing plasmids: 2721 with G17F12 and 2725 with G18A10. 


\section{Sterol mutants of $N$. crassa are pisatin-sensitive}

If the mechanisms underlying the nondegradative pisatin resistance were conserved, we would predict that sterol mutations in other fungi would also confer pisatinsensitivity. We tested this prediction using the erg mutants of $N$. crassa. The Neurospora erg mutations alter the composition of membrane sterols and confer nystatin resistance (Grindle, 1973) by blocking specific steps in ergosterol biosynthesis : erg 1 mutations affect $\Delta^{8,7}$ isomerization (Grindle \& Farrow, 1978), erg3 mutations affect the $\Delta^{14,15}$ reductase activity (Ellis et al., 1991). We found that, compared to the wild-type, the ergl and erg3 mutants were much more sensitive to growth inhibition on medium containing $40 \mu \mathrm{g}$ pisatin $\mathrm{ml}^{-1}$ (Table 4).

We used the pisatin-sensitive phenotype of the ergl and erg3 mutants to clone the respective genes by transforming mutant protoplasts with pooled plasmid DNA from the Orbach/Sachs ordered Neurospora genomic library (obtained from the FGSC) and selecting for pisatin-resistant transformants. The clones G17F12 and G18A10 were found to complement erg1 and erg3, respectively. The pisatin-resistant transformants had become nystatin-sensitive (Table 4), thereby suggesting that the complementing plasmids contained the corresponding wild-type alleles. A detailed molecular characterization of the cloned genes will be published elsewhere.

\section{Discussion}

Our results suggest that pisatin inhibits $D$. discoideum amoebae by two distinct inhibitory effects; one cytolytic and demonstrable even in non-growing cells and the other apparently cytostatic and demonstrable only under conditions favourable to cell growth. The $n y s C$ and the nys $B$ sunD mutants can acquire resistance to the cytolytic effect of pisatin, but they do not become resistant to the growth-associated inhibitory effect. Pisatin-resistant amoebae lose their resistance phenotype during incubation in BSS by first losing their resistance to the growth-associated inhibitory effect and then losing resistance to the cytolytic effect. The phenotype of the HK412 strain (viz. a partially constitutive resistance to the cytolytic effect but incapable of acquiring resistance to the growth-associated inhibitory effect) argues against the possibility that resistance to the two effects represents two thresholds in the continuum of a single mechanism. We propose that the induction of pisatin resistance involves the turning-on of independent mechanisms against the two inhibitory effects.

One of our original goals was to select pisatin-resistant mutants. We were unable to obtain such mutants by direct selections on SM-pis150 medium, even after mutagenesis with MNNG (frequency $<10^{-8}$ ). We can now account for our inability to obtain such mutants by our model that independent resistance mechanisms have to be turned-on for growth on SM-pis150, in which case it would be unlikely that a single gene mutation would confer the pisatin-resistance phenotype.

Mutants affected in nondegradative pisatin resistance have been reported, thus far, only in $D$. discoideum (Kasbekar \& Bhavani Prasanna, 1992; Kasbekar \& Papavinasasundaram, 1992). We have now demonstrated that in $N$. crassa, the ergl and $\operatorname{erg} 3$ mutations that alter sterol composition also conferred pisatin sensitivity. There is an important difference, however, between the pisatin-sensitivity phenotype of the erg mutants of $N$. crassa and that of the nys $C$ mutants of $D$. discoideum. The erg mutants are constitutively sensitive to a concentration of pisatin $\left(40 \mu \mathrm{g} \mathrm{ml}^{-1}\right)$ that is not inhibitory to the wild-type. In contrast, the pisatinsensitivity phenotype of the uninduced nys $C$ mutants is the same as that of the uninduced wild-type (Kasbekar \& Papavinasasundaram, 1992). Both grow equally well on SM-pis50 (the subinhibitory concentration of pisatin), and are equally sensitive on SM-pis 150 (the inhibitory concentration). The only difference between them was in their ability to acquire pisatin resistance during pretreatment with the sublethal concentration of pisatin; the wild-type was inducible whereas the nys $C$ mutants were blocked (Kasbekar \& Papavinasasundaram, 1992).

Our model that independent mechanisms are induced against the different inhibitory effects of pisatin can account for this difference in phenotype of $N$. crassa and $D$. discoideum sterol mutants on the assumption that, in $N$. crassa, a much higher concentration of pisatin is required for the cytolytic effect (possibly due to the presence of a cell wall) than for its growth-associated inhibitory effect. Consequently, at $40 \mu \mathrm{g} \mathrm{ml}^{-1}$ the wildtype remains viable, acquires resistance to the second effect, and only then resumes growth. The erg mutants cannot grow because they are blocked in the acquisition of resistance to the growth-associated inhibitory effect. In contrast, we suggest that in $D$. discoideum both the inhibitory effects occur at approximately the same pisatin concentration. Therefore, uninduced wild-type and $n y s C$ amoebae are equally sensitive to lysis at the inhibitory concentration $\left(150 \mu \mathrm{g} \mathrm{ml}^{-1}\right)$ and both are rapidly killed. After induction, the wild-type acquires resistance to both inhibitory effects but the mutants can acquire resistance to only the cytolytic effect. Hence the wild-type can grow after transfer to SM-pis150 but the mutant cannot. Thus in both systems, sterol alterations suppress the same mechanism, namely the growth-associated inhibition.

Preliminary studies (D. P. Kasbekar, unpublished) show that treatment with pisatin, together with the sterol biosynthesis inhibitor azasterol A25822B, completely blocked mycelial growth in the pea pathogen Nectria 
haematococca. At the concentrations used, pisatin alone was uninhibitory whereas the azasterol alone inhibited conidial development and retarded growth to some extent. This synergism of the action of pisatin with sterol change suggests that nondegradative pisatin resistance will be suppressed in erg-like mutants of Nectria. By examining the virulence of such mutants on pea, it should be possible to assess the role of this mechanism in pathogenesis.

We thank E. R. Katz for the nysB sunD double mutant HK412, Morris Grindle for the Neurospora strains, and J. Gowrishankar, Imran Siddiqi and Dipankar Chatterji for stimulating discussions and comments on the manuscript. We thank an anonymous referee for suggesting a key experiment that involved testing the fate of mutant cells on SM-pis150 medium. K.G.P. was supported by a post-doctoral fellowship from the Department of Biotechnology, Government of India. Some of the mutant selection experiments and studies on Neurospora and Nectria were done by D.P.K. during visits to the laboratories of H. D. VanEtten and M. J. Orbach on a Rockefeller Foundation Biotechnology Career Fellowship.

\section{References}

BONNER, J. T. (1947). Evidence for the formation of cell aggregates by chemotaxis in the development of the slime mold Dictyostelium discoideum. Journal of Experimental Zoology 106, 1-26.

DAvis, R. H. \& DE SERREs, F. J. (1970). Genetic and microbiological techniques for Neurospora crassa. Methods in Enzymology 28A, $79-143$.

Denny, T. P. \& VanEtren, H. D. (1983). Characterization of an inducible, nondegradative tolerance of Nectria haematococca MP VI to phytoalexins. Journal of General Microbiology 129, 2903-2913.

Denny, T. P., Matthews, P. S. \& VanEtten, H. D. (1987). A possible mechanism of nondegradative tolerance of pisatin in Nectria haematococca MP VI. Physiological and Molecular Plant Pathology 30, 93-107.

Ellis, S. W., Rose, M. E. \& Grindle, M. (1991). Identification of a sterol mutant of Neurospora crassa deficient in $\Delta^{14,15}$-reductase activity. Journal of General Microbiology 137, 2627-2630.

GRINDLE, M. (1973). Sterol mutants of Neurospora crassa: their isolation, growth characteristics and resistance to polyene antibiotics. Molecular and General Genetics 120, 283-290.

GRINDLE, M. \& FARRow, R. (1978). Sterol content and enzyme defects of nystatin resistant mutants of Neurospora crassa. Molecular and General Genetics 165, 305-308.

Kasbekar, D. P. \& Bhavani Prasanna, T. (1992). The nysB sunD double mutant of Dictyostelium discoideum is blocked in the acquisition of non-degradative resistance to the pea phytoalexin pisatin. FEMS Microbiology Letters 94, 251-254.

Kasbekar, D. P. \& Papavinasasundaram, K. G. (1992). An inducible, nondegradative phytoalexin resistance mechanism in Dictyostelium discoideum is suppressed by mutations that alter membrane sterol composition. Applied and Environmental Microbiology $\mathbf{5 8}$, 2071-2074.

Loomis, W. F. (1987). Genetic tools for Dictyostelium discoideum. Methods in Cell Biology 28, 31-65.

RAPER, K. B. (1935). Dictyostelium discoideum, a new species of slime mold from decaying forest leaves. Journal of Agricultural Research 50, $135-147$

SCandella, D., Rooney, R. \& Katz, E. R. (1980). Genetic, biochemical, and developmental studies of nystatin resistant mutants in Dictyostelium discoideum. Molecular and General Genetics 180, 67-75.

Sellitrennikoff, C. P. \& SAChs, M. S. (1991). Lipofectin increases the efficiency of DNA-mediated transformation of Neurospora crassa. Fungal Genetics Newsletter 38, 90-91.

Sussman, M. (1966). Biochemical and genetic methods in the study of cellular slime mold development. Methods in Cell Physiology 22, 397-410.

Sweigard, J. \& VANEtTEn, H. D. (1987). Reduction in pisatin sensitivity of Aphanomyces euteiches by polar lipid extracts. Phytopathology 77, 771-775.

Vollmer, S. J. \& YANOFSKY, C. (1986). Efficient cloning of genes of Neurospora crassa. Proceedings of the National Academy of Sciences of the United States of America 83, 4869-4873. 